\title{
OPTIMUM DESIGN OF COOLING PIPE SYSTEMS BY BRANCHING TREE MODEL IN NATURE
}

\author{
K. Yamazaki, ${ }^{1}$ and X. Ding ${ }^{2}$ \\ ${ }^{1}$ Dept. of Human \& Mechanical Systems Engineering, Kanazawa University, Kakuma-machi, \\ Kanazawa, 920-1192, JAPAN, yamazaki@t.kanazawa-u.ac.jp ${ }^{2}$ Dept. of Mechanical Engineer- \\ ing University of Shanghai for Science and Technology, 516 Jungong d.,Shanghai 00093, CHINA
}

\begin{abstract}
This paper suggests an innovative design methodology of heat transfer system based on a so-called adaptive growth law, which is an essential optimum growth rule of branch systems in nature. The branch systems in nature can grow by adapting themselves automatically to the growth environments in order to achieve better global functional performances, such as the maximal absorption of nutrition or sunlight in plants and the intelligent blood delivery of a vascular system in animal body. Thus, it can be expected that an optimum layout of heat transfer system would be obtained by the generation method based on the growth mechanism of branch systems in nature. First, the emergent process of branch systems in nature is reproduced in computer model by studying their common growth mechanisms. The branch systems are grown by the control of a so-called nutrient density so as to make it possible that the distribution of branches is dependent on the nutrient distribution. Then, the generation method is applied to the layout design problem for heat transfer systems. Both the conductive heat transfer system and the convective heat transfer system are designed by utilizing the generation method based on the growth mechanisms of branch systems in nature. The effectiveness of the suggested design method is validated by the FEM analysis and by the comparison with other conventional optimum design methods.
\end{abstract}

keywords: Layout Optimization, Cooling Channel, Branch System, Bionic Design

\section{Introductions}

Geometric forms (shapes and topologies) of branch systems in nature, such as lungs, vascular tissues, botanical tree (canopies, roots, leaves), etc., always show approximating a globally optimal performance that can minimize the costs of the construction and maintenance of the fluid transportation system under restraints of growth environment. So the branch systems are interested in modeling and visualizing not only by biologists but also by engineers. By studying the growth mechanisms of branch systems in nature, branch systems

Please use the following format when citing this chapter:

Author(s) [insert Last name, First-name initial(s)], 2006, in IFIP International Federation for Information Processing, Volume 199, System Modeling and Optimization, eds. Ceragioli F., Dontchev A., Furuta H., Marti K., Pandolfi L., (Boston: Springer), pp. [insert page numbers]. 
of plants and animals have been simulated by some kinds of approaches [1]- [4]. On the other hand, development of more intelligent and optimum engineering systems are expected by utilizing the optimality of branch systems in nature, and some effort have been done on this issue. For example, a topology design optimization method to generate stiffener layout pattern for plate and shell structures has been suggested [5], in which a growing and branching tree model is applied. The effectiveness of the method is proved because discrete stiffener layout pattern rather than a vague material distribution can be obtained.

In this paper, an innovative layout design methodology of heat transfer system by utilizing the optimality of branch systems in nature is suggested. The method bases on such essential characteristics of branch systems in nature that the branches can grow by adapting themselves automatically to the growth environments and achieve better global functional performances, such as maximal absorption of nutrition or sunlight in plants and intelligent blood delivery of a vascular system in animal bodies. Thus, it can be expected that an optimum layout of heat transfer system would be obtained by utilizing the generation method based on the growth mechanism of branch systems in nature. First, optimality and growth mechanisms of branch systems in nature are studied, and a reproduction approach of emergent process of branch systems is proposed. Branches are grown by the control of a so-called nutrient density so as to make it possible that the distribution of branches is dependent on the nutrient distribution. The growth of branches also satisfies the hydrodynamic conditions and minimum energy loss principle. If the so-called nutrient density in the generation process of branch system is referred to as the temperature in a heat transfer system, the distribution of branches is responsible to the distribution of cooling channels. Because branch system can grow adaptively corresponding to the nutrient distribution in order to absorb the nutrition to the maximal extent, the cooling channel can be constructed adaptively by the control of the temperature so as to make it possible to achieve comparative uniform temperature distribution of the whole heat transfer system. Having the similar optimality of branch systems in nature, the constructed cooling channel can be designed flexibly under any complex thermal boundary conditions within any shapes of perfusion volumes to be cooled and will achieve good cooling performance. The design problems of both the conductive heat transfer system and the convective heat transfer system are studied, and the cooling performances of the designed heat transfer systems are confirmed being improved by carrying out the FEM analysis and by comparing with the results designed by other conventional design methods. 


\section{Reproduction of Emergent Process of Branch Systems in Nature}

It is necessary to reproduce the emergent process of branch systems in nature in order to apply the optimality of branch systems in nature to engineering design, thus a generation approach of the emergent process for a hierarchical dichotomous branch system being considered as a material or energy transportation system is studied. A certain distributed nutrition density is assigned in advance in the perfusion space to control the growth of branches to make it possible that the distribution of branches is dependent on the distribution of the so-called nutrition density. During the generation process of branch system, the nutrition density in the whole perfusion space decreases by growing branches and its distribution tends to be uniform. The prerequisites of constructing such branch system are briefly described as follows:

1 Branching law stands for the relationship of radii between the parent branch and the daughter branches, which is adopted at every bifurcation point. For a dichotomous branch system, it is formulated as the following Eq. (1).

$$
r_{0}^{\lambda}=r_{1}^{\lambda}+r_{2}^{\lambda}
$$

where $r_{0}, r_{1}$ and $r_{2}$ are radii of the parent branch and the daughter branches, as shown in Figure 1. The bifurcation exponent, $\lambda$, is physiologically reasonable when it is in the range of $2 \leq \lambda \leq 3$. Murray's law shows the energy loss for transporting material throughout the whole network can be made minimum when $\lambda=3$.

2 Growing law relates to the growing direction and the growing velocity for a new branch, which is assumed to be dependent on the local nutrient distribution. A new terminal site is always positioned at the point with the highest nutrient density in the local growth space (vicinity) around the grown branches. If there is more than one such point in the local growth space, a point is selected by a pseudo random number sequence.

3 The hydrodynamic conditions are assumed as that each terminal branch has the same flow and pressure so as to bathe the whole perfusion space evenly. The branches are assumed to be cylinders. Flows in the branches are assumed as fully developed laminar flow, so they obey Poiseuille's law formulated by the following Eq.(2).

$$
Q=\frac{\pi r^{4}}{8 \nu} \frac{\Delta P}{l}
$$

where $Q$ is the volumetric flow rate, $\Delta P$ is the pressure drop, $r$ and $l$ are radius and length of the vessel, and $\nu$ is the dynamic viscosity of the fluid. 
4 The volume of whole branch system is selected as the cost function, so the branch system is designed in such a way that the volume of it is minimized,

$$
V=\sum_{i=1}^{n} r_{i}^{2} l_{i} \rightarrow \min
$$

where $r_{i}, l_{i}$ are radius and length of branch $i$, and $n$ is the total number of branches.

According to the above prerequisites, the growth process can be implemented as follows. A certain nutrition density distribution is assigned in a specified perfusion area in advance. The initial branches are grown, which satisfy the hydrodynamic conditions and a certain branching law. The local nutrition densities nearby the existent branches are updated. Then a new terminal site is selected at a point with the highest nutrition density in the growth area. If there is more than one point having the highest nutrition density, a candidate point is selected at random by a pseudo random number sequence. Therefore the density of branch distribution is dependent on the initial nutrition density of the perfusion area in order to absorb nutrition as much as possible. Next, the new terminal site is attached to the existent branches near it. For each attachable candidate branch, the bifurcation point is selected optimally with the objective of minimum volume of the whole network under the restraint of hydrodynamic conditions, and the new terminal site connects with the bifurcation point tentatively. The radius of the new branch is decided according to Poiseuille's law, and radii of all parents of the new segment are updated according to the branching law. Then the connection is dissolved but the volume of whole network for the connection is recorded. By comparing volumes of all possible tentative connected topologies, a connected topology with the minimum volume is finally adopted permanently. The growth process will stop when the average nutrition density in the perfusion area cannot be decreased anymore.

Figure 2 shows the simulation result of the growth process for a 2-dimensional branch system filled up a circular space. The initial nutrition density is distributed uniformly, as shown in Figure 2(a). Murray's law is adopted at each bifurcation. Figure 2(b)-(d) show the emergent process of the branch system, in which the total branch number of the finally generated branch system is 6971 . It is found that the branch system can fill up the whole specified space and the distribution of the branches is dependent on the initial nutrition density in the original space, which is almost uniform. During the generation process, a trunk grows at first, and some boughs grow spreading the whole perfusion area at very initial period. However, a number of twigs grow finally. The simulated branch system has both thicker and thinner size levels, which is similar to the branch systems in nature, in which the thicker ones convey long-distance material transportation, and the thinner ones exchange material with environment. 


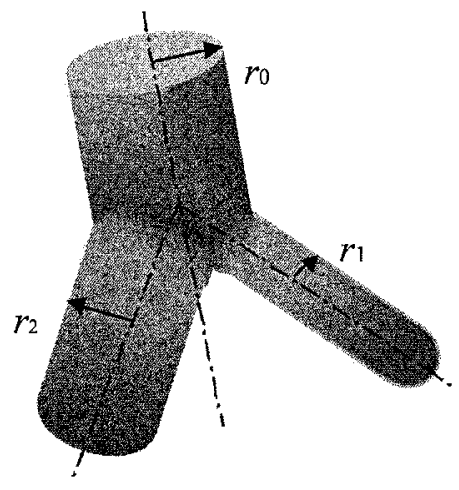

Figure 1. Geometry of a dichotomous branch system

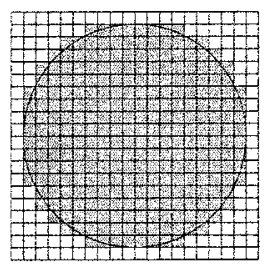

(a) Uniform nutrient density

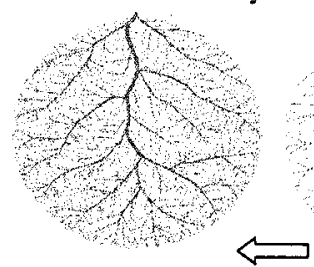

(d) Number of branch $=6971$

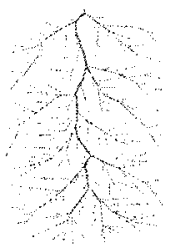

(b) Number of branch $=501$

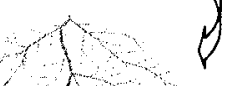

(c) Number of branch $=1001$

Figure 2. Growth process of branch system under uniformly distributed initial nutrition density ( $\lambda=3.0$, Volume rate $=1.60 \%$ )

If the circular perfusion area is considered as a plate with the thickness of $1 / 14$ of its radius, the volume rate of the branch system, i.e., the ratio of the branch volume to the whole plate volume, is $1.60 \%$.

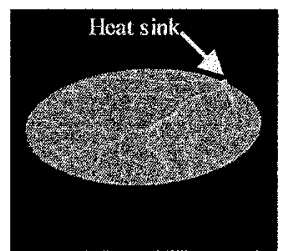

(a) FEM model (Volume rate $=9.71 \%$ )

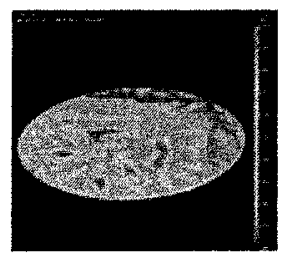

(b) Temperature field $T_{\max }=57.55^{\circ} \mathrm{C}$

Figure 3. FEM model and temperature field for a circular plate with a natural branch-like conductive cooling channel under uniform heat-generating rate

In conclusion, it is said that the suggested generation method can grow branch systems to fill up any specified perfusion areas with arbitrarily distributed initial nutrition densities, and the generated branch systems are qualitatively similar to the branch systems in nature. 


\section{Layout design of Heat Transfer System}

If the so-called nutrient density in the generation process of a branch system is referred to as the temperature in a heat transfer system, the distribution of branches can be considered corresponding to the distribution of cooling channels. Because the branch system can grow adaptively depending on the nutrient distribution in order to absorb the nutrition to the maximal extent, the cooling channel can be constructed adaptively by the control of the temperature so as to make it possible to achieve comparatively uniform temperature distribution of the whole heat transfer system. Therefore, the generation method based on the growth mechanism of branch systems in nature can be applied to the layout design of cooling channels in the heat transfer systems.

First, the layout of a conductive heat transfer system is designed and its cooling performance is analyzed. The problem is stated as: "a finite-size volume is to be cooled through a small patch (heat sink) located on its boundary, in which heat is being generated at every point. "A finite amount of high conductivity $\left(k_{p}\right)$ material is available. Determine the optimum distribution of material $k_{p}$ through the given volume such that the temperature distribution of the whole volume is as uniform as possible." The natural branch-like conductive cooling channel is constructed based on the corresponding original branch system, in which its cross-section is assumed to be a rectangle with the same thickness as the plate. The width of the channel, however, is assumed to be the same diameter as the corresponding branch in the branch system. The ratio of thermal conductivity of the high conductivity material $\left(k_{p}\right)$ to the low conductivity $\left(k_{0}\right)$ is assumed as $\tilde{k}=k_{p} / k_{0} \gg 1$. A circular plate with the ratio of thickness to diameter of 0.01 is considered as the finite-size volume to be cooled, in which the heat generates at every point with the uniform volumetric heat-generating rate $q^{\prime \prime \prime}=10^{5} \mathrm{~W} / \mathrm{m}^{3}$. The layout of the conductive cooling channel made of a high conductivity material $\left(k_{p}\right)$ is based on the original branch system shown in Figure 2(d), in which some branches with smaller cross-sections are omitted for the simplicity. It is noted that the distribution of the volumetric heat-generating rate is identical to that of the so-called nutrient density in the generation process of the original branch system, which is uniform as shown in Figure 2(a). The ratio of the thermal conductivities of the high conductivity material $\left(k_{p}\right)$ to the low conductivity $\left(k_{0}\right)$ is assumed to be $10^{4}$. The temperature at the heat sink located on the boundary is set as $T_{\min }=10^{\circ} \mathrm{C}$. The whole structure is insulated from the environment. Figure 3(a) shows the FEM model generated by some imaged section slide by Voxelcon. The volume rate of the cooling channel is $9.71 \%$. Figure 3(b) illustrates the temperature field of the whole plate, in which the red parts stand for the hot spots, while the blue parts stands for the comparative cool spots. The maximal temperature is $T_{\max }=57.55^{\circ} \mathrm{C}$. It is found that the hot spots are distributed over the whole volume. 
Next, the layout of a convective heat transfer system is designed by the generation method based on the growth mechanisms of branch systems in nature. The difference between the convective and the conductive heat transfer systems is that the coolant flow is available to remove the heat in the convective heat transfer system. The problem can be stated as: "construct an optimum convective cooling channel in a specified volume applied a certain distributed heat flux, in which coolant flows through the channel to remove the heat." A branch-like convective cooling channel is constructed inside a flat plate, which is based on the corresponding original branch system. The diameter of each segment in the cooling channel is assumed to be identical to that of the corresponding branch in the original branch system. A certain distributed heat flux is applied on the top surface of the plate, the bottom surface is insulated from the environment, and the circumference is on the forced air convection. Coolant with a certain volumetric flow rate is flown through the channel to remove the heat. The volume to be cooled is assumed to be made of beryllium-copper alloy, thermal conductivity of which is set as $k=260 \mathrm{~W} / \mathrm{mK}$. The coolant flowed through the cooling channels is assumed as water, and its temperature at inlet is assumed as $20^{\circ} \mathrm{C}$.

Actually, the problem is a transient heat conduction problem between the solid and coolant in the channel. However, because our goal here is only to confirm the heat conduction efficiency of the heat transfer system with a convective cooling channel, the problem is simplified as a steady-state heat conductive problem. However, it is necessary to consider the energy balance in the system responsible for the fluid convection. Because the temperature of coolant becomes higher and higher by passing through the cooling channel from inlet to outlet, the energy balance due to the fluid convection can be considered approximately as the distribution change of the temperatures at the channel wall. The temperature difference at the channel wall between the inlet and outlet of segment $i$ can be evaluated approximately by the following equation.

$$
\Delta T_{\mathrm{wi}}=\frac{q l_{\mathrm{i}}}{\rho_{\mathrm{c}} C_{\mathrm{c}} r_{\mathrm{i}} u_{\mathrm{ci}}}
$$

where $q$ is the heat flux applied on the channel, $\rho_{\mathrm{c}}$ and $C_{\mathrm{c}}$ are density, specific heat of coolant, respectively. And $u_{\mathrm{ci}}$ is the coolant average velocity of branch $i$.

Figure 4 shows the FEM models and temperature fields of three circular plates with branch-like convective cooling channels resulted from different branching laws. The branch-like convective cooling channels in the heat transfer systems are constructed based on the corresponding branch systems shown in Figure 2(d), in which the bifurcation exponents $\lambda$ are 3.0, 2.5 and 3.5, respectively. The thickness of each plate is assumed to be $1 / 14$ of its radius. The volumetric flow rate of coolant at the inlet is assumed $Q=8 \times 10^{-5} \mathrm{~m}^{3} / \mathrm{s}$, 
and the heat flux applied at the top surface of the plate is set as $q=5.0 \times 10^{5}$ $\mathrm{W} / \mathrm{m}^{2}$. The left figures in Figure 4 are the middle planes of the FEM models, in which the volume rates of cooling channels are $1.77 \%$ for $\lambda=2.5,1.28 \%$ for $\lambda$ $=3.0$ and $1.32 \%$ for $\lambda=3.5$, respectively. The right figures in Figure 4 show the temperature fields that are scaled by the maximal temperature $T_{\max }=44.67^{\circ} \mathrm{C}$ for $\lambda=2.5$. And the maximal temperatures are $T_{\max }=33.94^{\circ} \mathrm{C}$ for $\lambda=3.0$ and $T_{\max }=34.20^{\circ} \mathrm{C}$ for $\lambda=3.5$, respectively. It is easy to find that the case of $\lambda=3.0$ results the most uniform temperature distribution, and the most non-uniform one is the case of $\lambda=2.5$. It is noted that the volumes of the convective cooling channels from small to large is put in order as $\lambda=3.0,3.5$ and 2.5, which is the same sequence with the uniformities of temperature distributions. As a result, Murray's law $(\lambda=3.0)$ is the most effective branching law for constructing the branch-like convective cooling channel system. The reason is that Murray's law is derived from such principle that the energy loss due to the viscous friction throughout the whole network is minimal, that provides the easiest way for the coolant to pass.

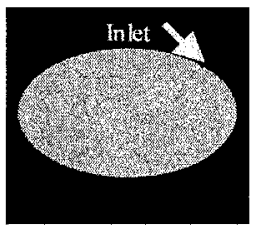

Vo hu me rate $=1.77 \%$

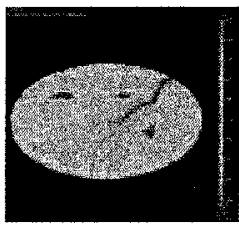

$T_{\max }=44.67^{\circ} \mathrm{C}$

(a) $\lambda=2.5$

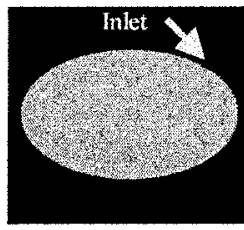

Volume rate $=1.28 \%$

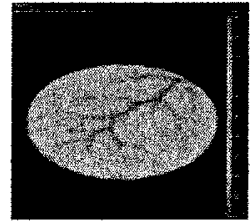

$T_{\max }=33.94^{\circ} \mathrm{C}$

(b) $\lambda=3.0$

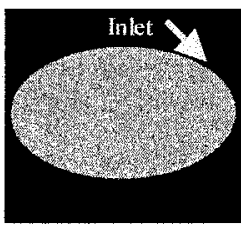

Volu me rate $=1.32 \%$

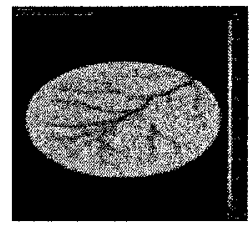

$T_{\max }=34.20^{\circ} \mathrm{C}$

(c) $\lambda=3.5$

Figure 4. FEM models and temperature fields for heat transfer systems with branch-like convective cooling channels resulted from different branching laws

In order to validate the effectiveness of the suggested generation method, the cooling performances between the natural branch-like cooling channel designed by the suggested generation method and the horizontal-vertical tree-like cooling channels constructed by the constructal theory proposed by Bejan [6] are compared. 
The unique principle of the constructal theory for designing the conductive cooling channel is: "every portion of the given volume can have its shape optimized such that its resistance to heat flow is minimal". By utilizing this principle, the cooling channel is determined in a sequence of steps consisting of shape optimization and subsequent construction. It starts from the smallest building block (elemental system) and proceeds toward larger building block (assemblies).

Figure 5(a) shows the FEM model and the corresponding temperature field of a horizontal-vertical tree-like conductive heat transfer system till second construction. The ratio of the thermal conductivities of the high-conductivity material to the low-conductivity material is assumed to be $\tilde{k}=k_{\mathrm{p}} / k_{0}=3333.33$. The volumetric heat-generating rate is set as $q^{\prime \prime \prime}=10^{5} \mathrm{~W} / \mathrm{m}^{3}$, and is distributed uniformly over the whole volume to be cooled. The volume is a square plate with the ratio of the thickness to the edge length 0.01 , which is derived from the sequence of shape optimization and construction by the constructal theory. The temperature at the heat sink end is set as $10^{\circ} \mathrm{C}$, and the whole structure is insulated from the environment. As shown in Figure 5(a), the volume rate of the cooling channel, i.e., the $k_{\mathrm{p}}$ material in which allocated in the whole volume is $9.47 \%$, and the maximum temperature is $T_{\max }=58.25^{\circ} \mathrm{C}$.

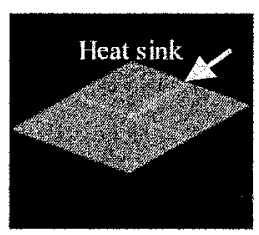

FEM model

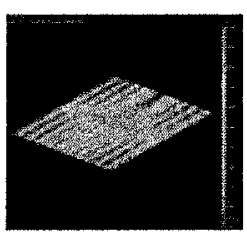

Temperature field

(a) Horizontal-vertical tree-like conductive

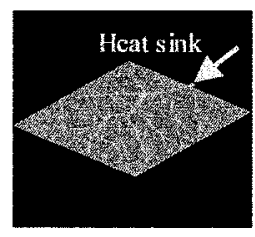

FEM model

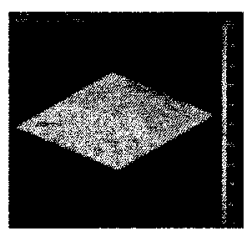

Temperature field

(b) Natural branch-like conductive cooling

Figure 5. Comparison of horizontal-vertical tree-like and natural branch-like conductive cooling channels 
According to the geometries of the heat transfer system with the horizontalvertical tree-like cooling channel, a corresponding branch system is generated on a square perfusion area applied a uniformly distributed nutrient density. Figure 5(b) shows its FEM model and the corresponding temperature field. The volume rate of the cooling channel is $9.62 \%$, which is very close to that of the horizontal-vertical conductive cooling channel. And the maximum temperature is $T_{\max }=53.32{ }^{\circ} \mathrm{C}$, which is a little lower than that of the horizontal-vertical tree-like cooling channel. It is found that hot spots are distributed over the whole volume to be cooled in both cases, so it is said that both conductive cooling channels can achieve good cooling performances. However, it should be noted it is just because the thermal boundary conditions are very simple (uniformly distributed heat-generating rates are applied), the simple and regular distributed conductive cooling channel, i.e., the horizontal-vertical tree-like conductive cooling channel, is available and effective. If the heat-generating rate is applied non-uniformly, it is difficult for the constructal theory to design effective conductive cooling channel, while the flexible natural branch-like conductive cooling channel can be designed adapting to the arbitrary complex thermal boundary conditions. Moreover, the design volume to be cooled can not be changed arbitrarily and be defined in advance when the constructal theory is adopted because of the definite time arrow of the construction from small to large. While the design volume can be defined in advance and the natural branch-like conductive cooling channel can be designed to fill up the volume with any shape by the suggested generation method. Therefore, it can be said that the suggested generation method is more powerful for designing the conductive cooling channel and the designed cooling channel can remove the heat generated in the matrix effectively.

\section{Conclusions}

By studying the growth mechanisms of branch systems in nature, an innovative layout design methodology of heat transfer systems is suggested in this paper. Having the similar optimality of branch systems in nature, the heat transfer system for cooling can be designed flexibly under any complex thermal boundary conditions within any specified shapes of perfusion volumes to be cooled, and can remove heat generated in the volume effectively. The effectiveness of the suggested design method has been validated by carrying out the FEM analysis and by comparing with other conventional design methods. It is expected that the suggested method can be applied to some more practical engineering applications, such as cooling channels in injection moulds, heat sinks in electronic packages, and so on. 


\section{References}

[1] H. Honda, Description of form of trees by the parameters of tree-Like body: effects of the branching angle and the branch length on the shape of the tree-like body, J. Theor. Bio. , 31, pp.331-338, 1971.

[2] A. Takenaka, A simulation model of tree architecture development based on growth response to local light environment, J. Plant Research, 107, pp.321-330, 1994.

[3] R. Takaki, H. Kitaoka, Virtual construction of human lung, Forma, 14, pp.309-313, 1999.

[4] W. Schreiner, R. Karch, F. Neumann, M. Neumann, Constrained constructive optimization of arterial tree models, In: Scaling in Biology, Edited by Brown, J.H. \& West G.B., Oxford university press, Inc., pp.145-165, 2000.

[5] X. Ding, K. Yamazaki, Stiffener layout design for plate structures by growing and branching tree model (application to vibration-proof design), Struct. Multidisc. Optim. , 26, pp.99-1 10, 2004.

[6] Bejan, A., Shape and structure, from engineering to nature, Cambridge, UK: Cambridge University Press, 2000. 